\title{
Neural classification maps for distinct word combinations in Broca's area
}

\author{
Marianne Schell ${ }^{1}$, Angela D. Friederici ${ }^{2}$, and Emiliano Zaccarella ${ }^{2, \mathbb{}}$ \\ ${ }^{1}$ Heidelberg University Hospital, Department of Neuroradiology, Im Neuenheimer Feld 400, 69120 Heidelberg, Germany \\ ${ }^{1} 1$ Max Planck Institute for Human Cognitive and Brain Sciences, Department of Neuropsychology, Stephanstraße 1a, 04103 Leipzig, Germany
}

\begin{abstract}
Humans are equipped with the remarkable ability to comprehend an infinite number of utterances. Relations between grammatical categories restrict the way words combine into phrases and sentences. How the brain recognises different word combinations remains largely unknown, although this is a necessary condition for combinatorial unboundedness. Here, we used functional magnetic resonance imaging and multivariate pattern analysis to explore whether distinct neural populations of a known language network hub —-Broca's area-are specialised for recognising distinct simple word combinations. The phrases consisted of a noun (flag) occurring either with a content word, an adjective (green flag), or with a function word, a determiner (that flag). The key result is that the distribution of neural populations classifying word combination in Broca's area seems sensitive to neuroanatomical subdivisions within this area, irrespective of task. The information patterns for adjective + noun were localised in its anterior part (BA45) whereas those for determiner + noun were localised in its posterior part (BA44). Our findings provide preliminary answers to the fundamental question of how lexical and grammatical category information interact during simple word combination, with the observation that Broca's area is sensitive to the recognition of categorical relationships during combinatory processing, based on different demands placed on syntactic and semantic processing. This supports the hypothesis that the combinatorial power of language consists of some neural computation capturing phrasal differences when processing linguistic input.
\end{abstract}

Correspondence: zaccarella@cbs.mpg.de

\section{Introduction}

When processing connected speech, our linguistic combinatorial capacity must be flexible enough to make sense of infinite word combinations (Ding et al., 2016; Sheng et al., 2019). Linguistic combination is, however, not an all-with-all process; words do not randomly combine but are constrained by systematic relationships between grammatical categories (Rizzi, 2012). A basic hypothesis to begin with is that the language combinatorial faculty consists of some neural computation that is sensitive to these relationships distinguishing different phrases. Understanding how different phrases are processed during linguistic combination in the mature brain complements studies of on the development of the combinatorial productivity in children (Yang, 2013), and the potential gap between human and non-human linguistic combinatorial capacities (Goucha et al., 2017). Word combination may refer to any kind of compositional process between words guiding the denotation of linguistic expressions in language (Heim, 1982; Heim and
Kratzer, 1998; Partee, 2010). Hemodynamic studies on word combination have traditionally focused on the comparison between sentences and pseudo-sentences versus unstructured word-lists used as non-combinatorial controls (Friederici et al., 2000a; Humphries et al., 2006, 2005; Jobard et al., 2007; Matchin et al., 2017; Mazoyer et al., 1993; Stowe et al., 1999; Zaccarella et al., 2017a; Zaccarella and Friederici, 2015). Meta-analytical examinations across studies revealed that word combination involves a large fronto-temporalparietal network, including Broca's area-pars opercularis (Brodmann Area, BA44) and pars triangularis (BA45) - in the inferior frontal gyrus (IFG); the inferior part of the posterior superior temporal sulcus/gyrus (pSTS/STG, BA22); the angular gyrus (AG, BA39); the middle/anterior temporal gyrus (MTG, BA21/BA22) and the temporal pole (TP, BA38; Zaccarella et al., 2017b). Within this network, the different regions appear to contribute to combinatorial processing depending on the nature of the linguistic information carried by the words forming the sequence. Neural assemblies in the middle/anterior temporal gyrus and in the angular gyrus have been shown to be sensitive to the amount of semantic information contained in the stimulus material-thus being specifically active during the processing of sentences containing real word semantics (Humphries et al., 2006, 2005; Pallier et al., 2011; Rogalsky and Hickok, 2009). Broca's area and the pSTG/STS appear to be conversely involved independently of semantic information for both real and jabberwocky sentences-e.g., the bluny telp is flamping the pouling toves-where content elements are replaced by pseudowords to engage syntactic processing through the remaining functional elements (Friederici et al., 2000a; Goucha and Friederici, 2015; Matchin et al., 2017; Pallier et al., 2011). As such, these regions appear sensitive to the amount of syntactic information within the linguistic stimulus.

Contrasting with the above observation, however, is the finding that Broca's region, and more specifically its most anterior part (BA45), has also been found to be modulated by semantic information when, for example, subjects are asked to evaluate the overall propositional meaning of the sentence (Newman et al., 2010; Zaccarella et al., 2017a; Zhu et al., 2009), or have to solve semantic ambiguities by selecting the appropriate word meanings within the sentence (Rodd et al., 2005; Vitello et al., 2014). Conversely, syntactic aspects of language processing appear to involve neural populations in BA44 in some studies (Friederici, 2011; Wu et al., 2019), 
or in BA45 in some other (Hagoort, 2005; Matchin et al., 2017; Matchin and Hickok, 2019; Pallier et al., 2011; Tyler and Marslen-Wilson, 2008). Studies testing single word processing and simple composition however seem to confirm a predominant role of BA45 for semantic processing-for example, local perturbation to the anterior portion of the IFG (along BA45) together with contemporaneous perturbation of the angular region has been found to influence semantic decisions on individual words, providing causal evidence that the anterior inferior frontal region is part of a semantic network including temporal-parietal regions, where the functioning of one area depends on the integrity of the other (Hartwigsen et al., 2016). A recent study focusing on simple phrases furthermore proposed that the involvement of BA45 for simple combinations reflects increased semantic load, as the region was active when comparing meaningful (fresh apple) or anomalous (awake apple) phrases with syntactically legal pseudo-phrases (fresh gufel; Graessner et al., 2021b). Conversely, when syntactic pseudo-phrases (this flirk) where contrasted with word-list sequences lacking syntactic information (apple flirk), BA44 was conversely found (Zaccarella et al., 2017a). As a matter of fact, an artificial grammar experiment reported that BA44, but not BA45, was maximally involved with the pSTS/STG when the grammaticality of artificial sequences had to be evaluated on the basis of the syntactic relations to be established between the elements of the sequence (Chen et al., 2021).

By this account, the major advantage of using simple two-word combination lies in the possibility to focus on the fundamental question of how lexical and grammatical information contribute to the most basic combinatory operations at the very basic two-word level in Broca's area, and to evaluate whether the selective involvement of BA44 and BA45 reflects the processing of distinct lexical categories forming the phrase. First electrophysiological and hemodynamic studies on simple linguistic processing have begun to provide initial evidence that word combination is differently represented on the cortex (Del Prato and Pylkkanen, 2014), and that within Broca's area, BA44 and BA45 are differentially active depending on the content or functional categories involved (Schell et al., 2017). An independent line of research has further shown that the strength of cortical entrainment tracking phrasal presentation rate during auditory language processing is sensitive to grammatical category information (Burroughs et al., 2021), thus suggesting that the human combinatorial capacity treats syntactic categories differently when processing linguistic input. This functional dissociation mirrors theoretical considerations indicating that words of different grammatical categories play distinct roles in the sentence (Baker, 2003; Rizzi, 2012). Content class categories-nouns, verbs, adjectives, and adverbs-primarily carry descriptive content by denoting entities and events expressed in the sentence. On the contrary, function words-determiners, conjunctions, prepositions, pronouns, and auxiliaries-principally provide grammatical features and purely formal relational properties.
As such, their role in language is to create the syntactic skeleton for grammatically correct sentences. Contentive and functional lexicon thus place different demands on syntactic and semantic processing in language. Empirically, early psycholinguistic investigations already suggested different underlying processing systems for contentive and functional categories, reflected in lower levels of accuracy for the processing of function words-irrespective of frequency-in the adult healthy populations (Bradley and Garrett, 1983) and in agrammatic patients (Friederici, 1985; Shapiro and Jensen, 1986), and in a later sensitivity to function words during language processing in children (Friederici, 1983). This differential behavioural pattern is supported by electrophysiological findings showing that the processing of the two vocabulary types correspond to different activity distributions across the cortex (Pulvermüller et al., 1995).

In the present study, we used simple two-word combinations to directly link neural responses in the IFG to basic phrasal combinations in a functional magnetic resonance imaging (fMRI) setting using multivariate pattern analysis as decoding method. Mimicking previous experimental designs on basic word composition (Del Prato and Pylkkanen, 2014; Schell et al., 2017) two phrasal types of equal syllabic length are used in the present experiment. A single noun (flag) could either form a noun phrase (NP) with an attributive adjective (green flag) or form a determiner phrase (DP) with a determiner (that flag) Note that here we use the DP notation to indicate the determiner + noun phrase, to illustrate the distinction between the two types of phrases investigated here. An introduction to the DP- vs NP-hypothesis for determiner + phrase constructions is provided in Bernstein, 2008, for the interested reader. Using a voxel-based classifier to capture the relationship between the spatial pattern of fMRI activity and experimental conditions, we searched for areas containing detailed information of category-specific phrasal processing in Broca's area neural populations. Such analysis is affine to theories of neural representation for population codes, where information is encoded in patterns of activity across large neuronal populations (Georgopoulos et al., 1986; Pouget et al., 2000). In the present case, we sought to understand how each voxel, within the independentlydefined anatomical maps for BA44/45, directly contributed to the classifiers. More specifically, which voxels contributed to classifying the phrases included in our DP sample as DP phrases and which voxels contributed to classifying the phrases included in our NP sample as NP phrases. We did this within progressively larger zones of prediction accuracy. We reasoned that, if Broca's area is sensitive to phrasal processing, it should first be able to classify phrasal combinations significantly above chance compared to a corresponding control region. Second, if the region is sensitive to phrasal differences, distinct neuronal populations inside the area should be recruited. In this case, spatial localisation should adhere to cytoarchitectonic subdivisions proposed for the region (Amunts et al., 1999). Here, we specifically expected neuronal populations associated with adjective + noun com- 
binations to be localised in BA45. Neuronal populations associated with determiner + noun combinations were expected to be localised in BA44. It was hypothesised that these effects would be independent of two manipulation tasks. In one case, participants were asked to perform a synonym judgment task, which has been used to test aspects of semantic processing in previous studies (Jefferies et al., 2009; Mollo et al., 2016). We refer to this task as the semantic task. In the other case they were asked to perform a word-class judgement task, which is instead used to test aspects of syntactic processing (Berkovitch and Dehaene, 2019; Friederici et al., 2000b). We refer to this task as the syntactic task. Evidence in favour of a functional subdivision in Broca's area would support the claim that Broca's area is associated with linguistic combination and that phrasal specificities might lie in the neuronal populations of BA 44 and BA45 (Zaccarella et al., 2017b).

\section{Materials and Methods}

Participants. Twenty-eight native German speakers (13 females and 15 males; mean age: 28.25 yrs., standard deviation 4.03) were recruited from an internal database and invited to participate twice in the study. All participants were right handed as assessed with the Edinburgh questionnaire(Oldfield, 1971), reported normal hearing and had normal or corrected-to-normal vision. None of them had any history of neurological or psychiatric disorder. The study was approved by the Research Ethics Committee of the University of Leipzig and followed the guidelines of the Helsinki declaration. Written informed consent was obtained from all participants according to the procedures approved by the $\mathrm{Re}$ search Ethics Committee of the University of Leipzig. All participants received monetary compensation after completing the experiment.

Experimental design. Our functional study manipulated two factors in a 2x2 factorial design (see Table 1). The first factor was PHRASE (NP: adjective + noun = "blue boot"; DP: determiner + noun = "this boot"). The second factor was TASK (SEM: "Word meaning" for the semantic task; SYN: "Word class" for the syntactic task).

Stimuli. The experiment was carried in German language. For the DP condition we used simple determiners followed by nouns with relative inflectional gender marking: dies +er (masculine)/+e (feminine)/+es (neutrum), "this"; jen -er/-e/es, "that"; jed -er/-e/-es "each", manch -er/-e/-es, "some". For the NP condition we used simple qualifying adjectives for the first phrase (lang -er/-e/-es, kurz -er/-e/-es, groß -er/e/-es, klein -er/-e/-es, English: long, short, big, small with their respective inflectional ending) and colour adjectives for the second phrase (rot -er/-e/-es, gelb -er/-e/-es, blau -er/-e/es, grün -er/-e/-es, English: red, yellow, blue, green). Each participant received a separate list of items from the set of all possible DP or NP combinations generated by combining determiners and adjectives with all pairs of synonyms obtained from the bidirectional rating (see SI Bidirectional Rating).

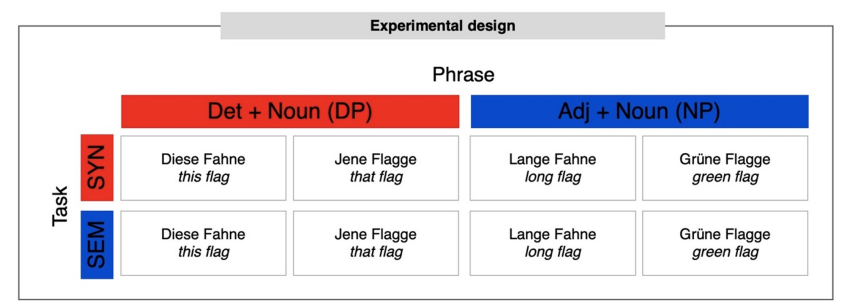

Table 1. Experimental design. The study crossed type of PHRASE and type of TASK in a $2 \times 2$ factorial design. The first factor was PHRASE (DP: determiner + noun = "this flag"; NP: adjective + noun = "green flag"), the second factor was TASK (SYN: "Word class" for the syntactic task; SEM: "Word meaning" for the semantic task).

Items were matched for syllabic length and controlled for bigram frequency using the google web1t database containing n-gram counts for approximately 100 billion word-tokens for the German language (Linguistic Data Consortium, University of Pennsylvania). Average bigram frequencies (log) across participants were 2.28 (SD: 0.10) for the DPs and 2.12 (SD: 0.10) for the NPs, with an averaged effect size (r) of 0.018 (C.I. =-0.14-0.17; see Fig. S6) (Sassenhagen and Alday, 2016).

Procedure. A trial always consisted of pairs of spoken twoword phrases (see SI Audio Recordings). The noun of the second phrase was either a repetition, a synonym, or an unrelated noun of the noun in the first phrase (see Stimuli). Bidirectional ratings of the semantic similarities of noun pairs were performed (see SI Bidirectional Rating). Each experimental block started with an instruction screen ("Word meaning" or "Word class") and consisted of a sequence of 12 trials. Each block always contained 3 DP trials, 3 NP trials, 3 mixed trials, and 3 filler trials in randomised order. Blocks were presented on a grey screen using black or white letters and symbols. In the semantic task, participants were asked to judge whether the second phrase could refer to the same object as described in the first phrase of the trial, by pressing the button box placed inside the scanner. In the syntactic task, participants judged whether the second phrase consisted of the same word class elements as the ones contained in the first phrase of the trial. The presentation of each trial was time-locked with volume acquisition, which is indicated as beneficial for multivariate analyses (Etzel et al., 2009). We split the whole experiment into two experimental sessions on two separate days lasting $28 \mathrm{~min}$ each. Each session contained 144 trials in twelve alternating task blocks. On each day participants performed 40 trials of each task outside the scanner to get familiar with the experimental setup. Training stimuli were not used inside the scanner. The experiment was implemented in Presentation software package (Neurobehavioral Systems, Inc., Berkeley, CA, https://www.neurobs.com/).

Data acquisition. MRI data were obtained using a 3-Tesla Siemens Magnetom Prisma scanner (Siemens Medical Systems, Erlangen, Germany) with a 20-channel head coil at the Max Planck Institute for Human Cognitive and Brain Science in Leipzig, Germany. To reduce fatigue the functional images were acquired on two separate days, in total, $1554 \mathrm{~T} 2 *$ 
weighted echo-planar images (777 images for each scan day, $\mathrm{TR}=2110 \mathrm{~ms}, \mathrm{TE}=22 \mathrm{~ms}$ ). Each volume consisted of $40 \mathrm{ax}-$ ial slices, parallel to AC-PC line, in ascending direction, and with whole brain coverage. Scans had an in-plane resolution of $3 \times 3 \mathrm{~mm}$ and a $2.5 \mathrm{~mm}$ slice thickness with an inter-slice gap of $0.5 \mathrm{~mm}$ (flip angle $90^{\circ}$, field of view $192 \mathrm{~mm}$, matrix size $64 \times 64)$.

Behavioral data analysis. Behavioural data were analysed using SPSS version 22.0 (SPSS, Inc., Chicago LI). Task performance was assessed by calculating accuracy and reaction times across trails using the response of the first scanning day, since the button presses could not be registered during the second scanning day for some subjects due to a technical error in the behavioural recording system. These participants had performed at ceiling during the first scanning day and self-reported to have accomplished the task without any difficulty on the second scanning day. Participants with an overall accuracy below $80 \%(\mathrm{~N}=5)$ were excluded from the further analysis. One additional participant was excluded due to excessive movement in the scanner (see below). Twentytwo participants ( 11 females and 11 males; mean age of 27.86 yrs., standard deviation of 3.99) were included in the analysis of the imaging data.

Imaging data preprocessing. Preprocessing was conducted with SPM8 (Wellcome Imaging Department, University Collage, London, UK, https://fil.ion.ucl.ac.uk/spm/) as implemented in MATLAB 7.14.0.739 (R2012a, Mathworks, Inc., Sherborn, MA, USA, https://de.mathworks.com). For each day, functional images were realigned to the first image and unwrapped with an accompanying fieldmap and corrected for movement induced variance by rigid and non-rigid transformation. Subsequently, the functional images were slice time corrected with the middle slice as a reference, coregistered to participants' individual T1-weighted image, acquired in a previous session, and normalised to MNI space using unified segmentation with a resampling of the images into $3 \mathrm{~mm}$ isotropic voxels. The 5 initial volumes were excluded to allow for magnetic saturation effects. The unsmoothed images were handed to the decoding toolbox for multivariate analysis to preserve fine-grained subject-specific information pattern(Kamitani and Sawahata, 2010).

First level analysis. In order to investigate the neural encoding of the trial types, a General Linear Model (GLM) was estimated using 3 runs of normalised data for each day, resulting in 6 runs for the whole experiment. Each run comprised 4 task blocks, 2 of each task, with 6 trials per condition, so that the experiment was fully balanced between the runs. A regressor for each condition and each run was entered. All regressors were locked to the onsets of the trial, while the durations were set to cover the full length. We added the movement parameters as separate regressors of no interest to the model. The resulting 24 beta images ( 6 runs $* 4$ conditions) were used for the multivariate pattern analysis.
Multivariate pattern analysis. We used "The Decoding Toolbox", TDT(Hebart et al., 2014) to determine the decision boundary for the different conditions within the data. The toolbox implements LIBSVM software (Chang and Lin, 2011) to train a linear support vector machine (SVM) with a decision function of a fixed cost parameter, $\mathrm{c}=1$. We applied two different classification approaches, an ROI method to evaluate Broca's area and a searchlight method for wholebrain classification. Both approaches used a stratified, leaveone-run-out, cross-validation ( 6 folds by 6 times classification) always using 5 runs for the training set ( 20 beta images, 10 for each class) and the "left-out" 6th run to evaluate the data ( 4 beta images, 2 for each class) and containing novel items of the same conditions. This is repeated several times as a function of the numbers or runs included in the study and averaged afterwards to obtain a mean decoding value of informativity, that allows inferences about the representational content of a certain region (Haynes and Rees, 2006; see also Fig. S5). The first classification was done alone with the PHRASE factor to see which patterns were able to classify participants as listening to DPs or to APs. We assigned all DPs-irrespective to task - to class +1 and all APs-again, irrespective to task- to class -1 . Here, class number refers to the direction of the binary decision during the classification processing. The second classification was done along the TASK factor. Here, all trials of the syntactic task were assigned to class +1 and all trials for the semantic task were assigned to class -1 . Threshold for significance was set at $\mathrm{p}<$ 0.05 .

Broca's area decoding analysis. Broca's area (see Fig. S1) was composed using the left area 44 (BA44) and area 45 (Amunts et al., 1999) (BA45) from the SPM Anatomy toolbox v 1.8 (Eickhoff et al., 2005) and assessed via the GUI. The left primary visual cortex (V1, BA17) from the same toolbox was used as control region to validate the classification approach (Etzel et al., 2009). The images were coregistered so that the number of voxels used for the classification analysis was similar across the participants (IFG with 421 voxels, V1 with 400 voxels). For each participant and classification, we extracted: one accuracy value per ROI and several SVM pattern values, one for each voxel within the ROI (see below). Both were determined by averaging the values across the folds.

Broca's area classification accuracy. For both Broca's area and V1 we calculated the classification accuracy. A one sampled t-test was used to calculate the significance of the classification against the chance level of 50\%. A second paired t-test was performed to test whether the classification in Broca's area performed better than in V1. We additionally analysed the mean specificity for each class separately to assess any asymmetry in classifier behaviour via two-sided paired t-tests.

Broca's area classification maps. While the accuracy refers to the success rate of the classification, SVM pattern determines the contribution of a voxel to the final clas- 
sifier function-also implemented in TDT toolbox-to get the positive and negative distance of the separating hyperplane(Haufe et al., 2014). High positive values for a voxel corresponded to high contribution to classify a stimulus as being a DP (or syntactic TASK). Similarly, high negative values for a voxel corresponded to high contribution to classify a stimulus as being an NP (or semantic TASK). All SVM pattern values were averaged across participants, resulting in one value per voxel. As the voxels with the largest magnitude contribute the most to the classification results, we first selected the $5 \%$ (21 voxels), 10\% (42 voxels) and 15\% (63 voxels) of largest positive and negative values for both classification analyses. We then obtained the classification maps by localising the same voxels within BA44 and BA45 to assess the distribution of the SVM pattern for the different conditions in Broca's area. Statistical significance of the observed voxel distribution was tested with a $\chi^{2}$-test. A Pearson correlation coefficient between condition and class classification, was also computed on the corresponding SVM patterns obtained from the maps described above to obtain a measure of Broca's area correlational specificity.

Unselective voxels in Broca's area. We searched for unselective voxels showing strong classification patterns for both DP phrase (independent of task) and for syntactic task (independent of condition). Similarly, we searched for unselective voxels exhibiting strong classification patterns for both NP phrase (independent of task) and semantic task (independent of condition). Unselective voxels within Broca's area were detected by combining the classification maps for PHRASE and TASK classification, which we then back-localised to BA44 and BA45. Since the overlapping degree at 5\% was barely manifest and only ranged between $0<\mathrm{N}_{\text {VOXELS }}<3$ across conditions, we restricted our analysis to the best $10 \%$ and $15 \%$ of the coding voxels. Fisher's Exact tests were used to assess the distribution between unselective voxels (semantics/syntax) and area (BA44/45). We further calculated percentiles based on the probability of the overlapping voxels estimated through bootstrapping with 1 million samples.

\section{Whole-brain searchlight analysis for PHRASE and} TASK classifications. Whole-brain analyses were performed to create overall information maps to show the pattern differences across the brain for both PHRASE and TASK classification. Thereby, accuracy values were assigned to the central voxel within each spherical searchlight of $12 \mathrm{~mm}$ (equals 4 voxels), iteratively performed over all voxels in the brain, resulting in a 3-dimensional accuracy map. Group analysis was performed by combining the individual subject maps with a t-test at each voxel as implemented in SPM8 to evaluate the classification approach (see Fig. S5).

\section{Results}

Behavioural data results. Findings from twenty-two German native speakers are reported. The average accuracy rate for the DP condition during syntactic task (DP-SYN) was 0.95 (standard deviation, SD: 0.07) and it was 0.95 (SD:
0.08) during the semantic task (DP-SEM). The average accuracy rate for the NP condition during the syntactic task (NP-SYN) was 0.96 (SD: 0.06) and it was 0.98 (SD: 0.03) during the semantic task (NP-SEM). We performed a $2 \times$ 2 within-subject design ANOVA with factors condition (DP and NP) and task (SYN and SEM). The analysis revealed a some tendency towards significance for the effect of condition $\left(\mathrm{F}_{(1,21)}=3.26 ; \mathrm{p}=0.08\right)$, no effect of task $\left(\mathrm{F}_{(1,21)}=0.55\right.$; $\mathrm{p}=0.47)$ and no interaction $\left.\mathrm{F}_{(1,21)}=0.44 ; 0=0.51\right)$ at a threshold of $\mathrm{p}<0.05$. The average reaction times of the DP condition during syntactic task (DP-SYN) was $848.3 \mathrm{msec}$ (standard deviation, SD: $307.1 \mathrm{msec}$ ) while it was $709.7 \mathrm{msec}$ (SD: $222.8 \mathrm{msec}$ ) during the semantic task (DP-SEM). The average reaction times for the NP condition during the syntactic task (NP-SYN) was $815.7 \mathrm{msec}$ (SD: $306.1 \mathrm{msec}$ ) and $710.5 \mathrm{msec}$ (SD: $253.3 \mathrm{msec}$ ) during the semantic task (NP-SEM). This $2 \times 2$ within-subject ANOVA showed a significant difference of reaction time for the tasks $\left(\mathrm{F}_{(1,21)}=17.502\right.$; $\left.\mathrm{p}<0.05\right)$, indicating that the syntactic task was slightly more difficult than the semantic task. The main effect of phrase $\left(\mathrm{F}_{(1,21)}=0.76\right.$; $\mathrm{p}=0.393)$ and the interaction $\left(\mathrm{F}_{(1,21)}=0.373 ; \mathrm{p}=0.548\right)$ showed no significant effect.

Broca's area classification accuracy. We first evaluated the accuracy for Broca's area (see Fig. S1) in comparison to control region (V1) for PHRASE and TASK classification. Single overall classification accuracies are reported in Fig. 1. Both PHRASE and TASK classifications revealed significant accuracy differences compared to the control region in $\mathrm{V} 1$ and chance level set at 50\% (PHRASE: IFG against V1: $\mathrm{t}_{(21)}=3.712 ; \mathrm{p}=0.001 ;$ IFG against $50 \%$ : $\mathrm{t}_{(21)}=5.328 ; \mathrm{p}<0.001$; TASK: IFG against $\mathrm{V} 1: \mathrm{t}_{(21)}=3.239$; $\mathrm{p}=0.004$ IFG against $\left.50 \%: \mathrm{t}_{(21)}=4.812 ; \mathrm{p}<0.001\right)$. No imbalance in the classifier behaviour was found: $\mathrm{p}=0.141$, $\mathrm{t}_{(21)}=1.530$ for the classification between conditions-DP classification of $63.64 \%$ ( $\mathrm{SD}=11.37 \%$ ), NP classification of $67.58 \%(\mathrm{SD}=14.74 \%)$, and $\mathrm{p}=0.254, \mathrm{t}_{(21)}=1.173$ for classification between the tasks-syntactic task accuracy was $67.42 \%(\mathrm{SD}=15.19 \%)$, semantic task accuracy was $62.50 \%$ $(\mathrm{SD}=19.71 \%)$.

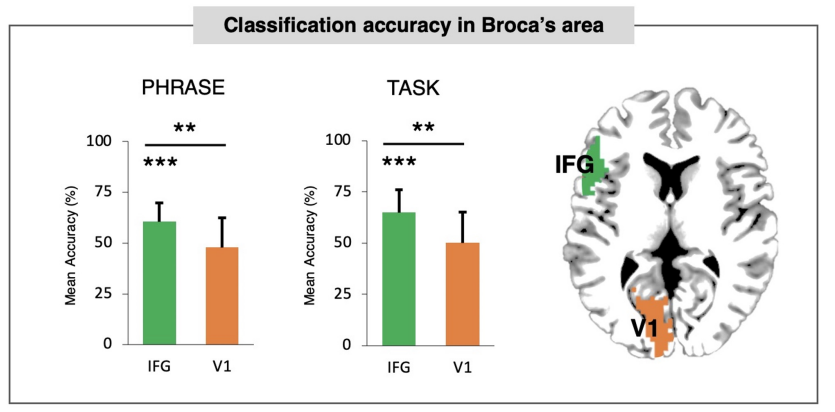

Fig. 1. Classification accuracy in Broca's area. PHRASE classification and TASK classification accuracy for Broca's area tested against control region (V1/BA17). Both PHRASE and TASK classifications in Broca's area revealed significant accuracy differences compared to both control region and chance level set at $50 \%$. Bars denote Standard Deviation (SD). IFG, inferior-frontal gyrus. Bars denote standard deviations. ${ }^{* *} \mathrm{p}<0.005,{ }^{* * *} \mathrm{p}<0.001$. Figure created using the MultiImage Analysis GUI viewer (Mango, Version 4.1; http://ric.uthscsa. edu/mango/) and MATLAB 7.14.0.739 (R2012a, Mathworks, Inc., Sherborn, MA, USA). 
Broca's area classification maps. We then asked whether voxel contribution to each specific class followed the cytoarchitectonic organisation of area 44 and 45 within Broca's area. We used the SVM pattern values (Haufe et al., 2014) to show the contribution of each voxel to the representation of the class under analysis. We chose 5\%, 10\%, and $15 \%$ of the highest positive and negative values corresponding to the most discriminative voxels for each classification. Finally, we assigned each of the selected voxels back to either BA44 or BA45, to assess the distribution of the SVM pattern for the different conditions (See Fig. 2). For the PHRASE classification $\chi^{2}$-tests showed significantly distinct distributional patterns in Broca's area, with voxels identifying determiner + noun combinations being strongly located in BA 44 . Voxels classifying adjective + noun combinations were conversely located in BA 45 $\left(5 \% \chi^{2}(1)=6.11, \mathrm{p}=0.013 ; 10 \% \chi^{2}(1)=17.28, \mathrm{p}<0.001 ; 15 \%\right.$ $\left.\chi^{2}(1)=23.52, p<0.001\right)$. Cramèr's $V$ magnitude of relationship test(Rea and Parker, 1992) revealed a relatively large association strength between the type of phrase and anatomical region for three subset distributions $\left(\mathrm{V}_{5 \%}=0.38 ; \mathrm{V}_{10 \%}\right.$ $=0.45 ; \mathrm{V}_{15 \%}=0.42$; see Fig. 3). This indicates that the type of condition had a strong effect on whether the location was in BA44 or BA45. For the TASK classification, $\chi^{2}$-tests revealed significant distributional patterns for the same regions $\left(5 \% \chi^{2}(1)=7.78, \mathrm{p}=0.0053 ; 10 \% \chi^{2}(1)=8.05, \mathrm{p}=0.0045\right.$; $\left.15 \% \chi^{2}(1)=8.18, \mathrm{p}=0.0042\right)$, confirmed by the moderate association strength between type of task and anatomical region $\left(\mathrm{V}_{5 \%}=0.43 ; \mathrm{V}_{10 \%}=0.30 ; \mathrm{V}_{15 \%}=0.25\right.$; Fig. $\left.\mathrm{S} 2\right)$. We further tested whether there was a linear relationship between PHRASE and TASK classifications to see if the voxels most informative for one classification where also the most informative for the other classification. While the correlation coefficient confirmed a small, positive, but significant relation between the voxels (Pearson: $\mathrm{r}=0.135, \mathrm{p}=0.005$ ), the $\mathrm{R}^{2}$ of 0.018 suggests that only $1.8 \%$ of the variance within the data was explained. This indicates that distinct neural populations underlie the coding of different conditions (see Fig. S3).

Unselective voxels in Broca's area. We then looked at intraregional information in BA44 and BA45 to determine the orthogonal overlap between PHRASE and TASK classification, to inspect the distribution of unselective voxels within the area. We therefore selected voxels showing strong classification for both DP phrase (independent of task) and for syntactic task (independent of condition). Similarly, we searched for voxels exhibiting strong classification patterns for both NP phrase (independent of task) and semantic task (independent of condition) within the $10 \%$ and $15 \%$ analyses. The $10 \%$ analysis yielded 10 unspecific voxels for syntax and 10 for semantics These voxels showed the following distribution: AREA(TASK $\cap$ CONDITION $)=\mathrm{N}_{\text {VOXELS }}(\%)$ : $\mathrm{BA}_{4} 5_{(\mathrm{SEM} \cap \mathrm{NP})}=8(88.88 \%) ; \mathrm{BA} 45_{(\mathrm{SYN} \cap \mathrm{DP})}=1(11.11 \%) ;$ $\mathrm{BA}_{4} 4_{(\mathrm{SEM} \cap \mathrm{NP})}=2(18.18 \%) ; \mathrm{BA} 44_{(\mathrm{SYN} \cap \mathrm{DP})}=9(81.81 \%)$. Fisher's Exact Test was significant at $\mathrm{p}=0.006$. The $15 \%$ analysis yielded 17 unspecific voxels for syntax and 17 for semantics with the following distributions: BA45 (SEMกNP) $=14(82.35 \%) ; \mathrm{BA} 45_{(\mathrm{SYN} \cap \mathrm{DP})}=3(17.64 \%) ; \mathrm{BA} 44_{(\mathrm{SEMNP})}=3$

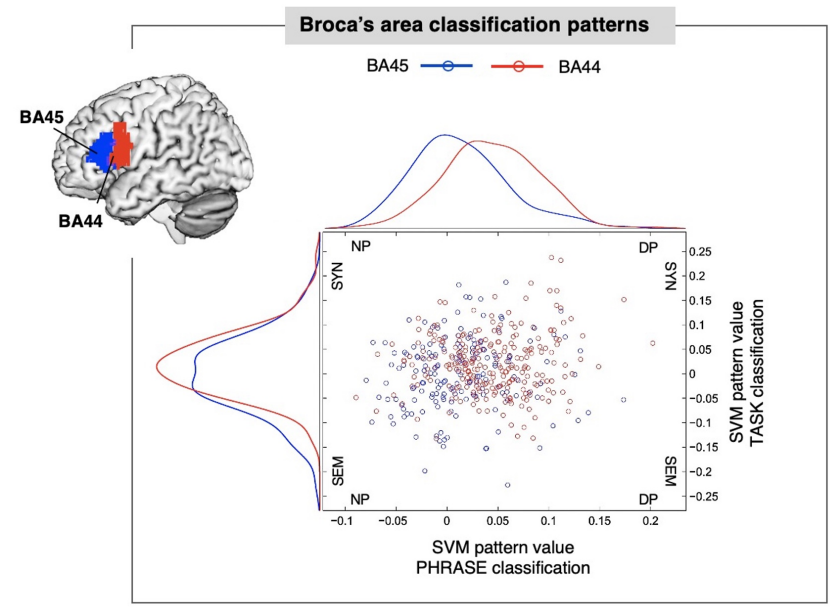

Fig. 2. Broca's area classification patterns. SVM (support vector machine) pattern values within the anatomically-defined Brodmann Area (BA) 44 and BA 45 of Broca's area (top-left), expressing positive and negative distances of each voxel from the separating hyperplane for PHRASE and TASK. Blue dots represent voxels located in BA45. Red dots represent voxels located in BA44. Curves represent pattern distribution for PHRASE and TASK in BA44 and BA45, respectively. SYN, syntax; SEM, semantics; NP, noun phrase; DP, determiner phrase. Figure created using the Multi-Image Analysis GUI viewer (Mango, Version 4.1; http://ric. uthscsa.edu/mango/) and MATLAB 7.14.0.739 (R2012a, Mathworks, Inc., Sherborn, MA, USA).
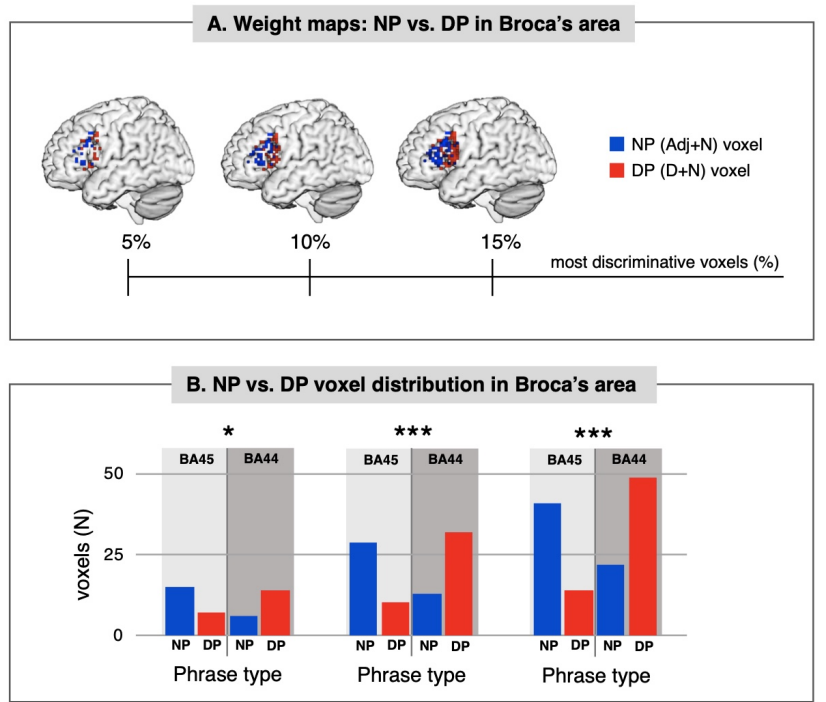

Fig. 3. PHRASE classification patterns. (A) Weight maps: NP vs. DP in Broca's area. The most discriminative $5 \%, 10 \%$ and $15 \%$ of classifier weights from the classifier trained to discriminate DP vs. NP, along the PHRASE factor in Broca's area. (B) NP vs. DP voxel distribution in Broca's area. PHRASE classification $\chi^{2}$-tests showed significantly distinct distributional patterns in Broca's area for the $5 \%$ (left), $10 \%$ (middle) and 15\% (right) most discriminative voxels, with voxels identifying determiner + noun combinations being strongly located in BA 44, while voxels classifying adjective + noun combinations being conversely located in BA 45. NP, noun phrase; DP, determiner phrase. ${ }^{*} p<0.05,{ }^{* * *} p<0.001$. Figure created using the Multi-Image Analysis GUI viewer (Mango, Version 4.1; http://ric.uthscsa.edu/mango/) and MATLAB 7.14.0.739 (R2012a, Mathworks, Inc., Sherborn, MA, USA)

$(17.64 \%) ; \mathrm{BA} 44_{(\mathrm{SYN} \cap \mathrm{DP})}=14(82,35 \%)$. Fisher's Exact Test was significant at $\mathrm{p}<0.001$. For both analyses, unselective syntactic voxels were localised mainly in the dorsal section of BA44, while unselective semantic voxels were conversely mainly localised in the posterior-ventral section of BA45 (see Fig. S4). The number of unselective voxels for both $10 \%$ (N 
$=20)$ and $15 \%(\mathrm{~N}=34)$ analyses fell above the 99.99th percentile of our estimated distribution, meaning that nearly all the 1 million bootstrap samples had fewer overlapping voxels.

\section{Whole-brain searchlight analysis for PHRASE and} TASK classifications. Whole-brain analyses were finally performed to create overall information maps across the brain for both PHRASE and TASK factors. For the PHRASE classification, the information map on the group level showed a broadly distributed network mainly located in the left hemisphere. This included the superior and middle temporal gyri (MTG/STG), angular gyrus (AG), supramarginal gyrus (SMG) and inferior frontal gyrus (IFG). For the TASK classification the information map showed distributed spatial information patterns in the left and right IFG and in the left AG (see Fig. S5).

\section{Discussion}

The key finding of this study is that neural populations classifying simple word combinations in Broca's area, a high-order hub of the linguistic system that governs morphosyntactic relationships among words (Goucha and Friederici, 2015; Musso et al., 2003; Pallier et al., 2011), are not uniformly distributed. Rather, classification involves neuroanatomically distinct sub-regions within the area, reflecting a distinct neural processing for the different phrases included in our experimental sample.

The present study shows that Broca's area is sensitive to the recognition of distinct word combinations and that the recognition specificity lies in distinct neuronal populations of BA44 and BA45. Anterior Broca's area (BA45) contains a significantly larger number of voxels than posterior Broca's area (BA44) in identifying phrases formed by adjective + noun combinations. Given the rich conceptual content of the adjective + noun classification, we believe that these findings are in agreement with both correlational and causal analyses of semantic load in this area (Graessner et al., 2021; Hamilton et al., 2009; Hartwigsen et al., 2016). Conversely, the observation that posterior Broca's area contains a significantly larger number of voxels that are informative for the recognition of determiner + noun combinations with reduced conceptual content, appears to mirror syntactic processing effects found in the area for longer structures, regardless of meaning (Bornkessel et al., 2005; Chen et al., 2021; Friederici and Kotz, 2003; Goucha and Friederici, 2015; Zaccarella et al., 2017a). The identification of a set of voxels in the ventral region of BA45 that are responsive to both adjective + noun classification and the semantic task classification fits with the proposal of the anterior Broca's area as a generalised executive semantic processor (Chiou et al., 2018). Conversely, the set of voxels found in dorsal BA44, responsive to both determiner + noun and syntactic task classification, are consistent with findings showing that the dorsal region, but not ventral, is specifically active when syntactic complexity evaluation interfaces with domain-general mechanisms (Amici et al., 2007; Makuuchi et al., 2009).

Overall, Broca's area sensitivity to linguistic sequences is functionally consistent with neural activity measured in the area that correlates with the sound envelop of sentence structures prior to verbalization (Magrassi et al., 2015), or during reading (Nelson et al., 2017). Recently, the investigation of specific time scales for the processing of linguistic units and their neural signatures has shown that the brain is able to track linguistic (phrasal, sentential) dimensions not readily available in the input (Ding et al., 2016; Sheng et al., 2019). The strength of the cortical tracking, however, seems to be modulated by the kind of grammatical category included in the stimulus. This suggests that word-level grammatical information is taken into account to build larger units (Burroughs et al., 2021). In this cited study, in fact, entrainment seems to be significantly reduced when the linguistic stimuli contain phrases formed by different grammatical categories, compared to when the stimuli contain the same grammatical category occurring in a strictly alternating fashion. Much in the same spirit, our work begins to look at the representation patterns of different phrases, complementing previous neurobiological approaches to classify types of linguistic combinations on the cortex (Artoni et al., 2020).

The current findings leave open a few questions. First, here we used a well-controlled, but relatively small sample of determiners and adjectives that were nonetheless combined with a large sample of nouns. This was to reduce inherent baseline issues due to the contrastive nature of the methodology we used (Stark and Squire, 2001), notwithstanding the use of a total number of items similar to other fMRI studies on language employing MVPA techniques (Herrmann et al., 2012). As such, our classifiers were not tested on novel determiners and adjectives that were not included in our stimuli samples, which lessens the generalisability of the present results to more abstract category-based distinction. At the same time, however, the nature of our block design, in which words were never heard in isolation but always as two-word phrases, makes it difficult per se to draw conclusions about the effects of individual words and category types. Rather, our work provides preliminary evidence that the brain processes incoming phrases differently, depending on the amount of syntactic and semantic information included during the combinatorial process. Emerging new decoding methods, along different levels of abstraction (Wurm and Lingnau, 2015) and indications from computational approaches (Hewitt and Liang, 2019), may help to prove whether our findings can generalize to other members of each category in Broca's area. Second, we concur with the idea that linguistic theories make representational hypothesis about the combinatorial mechanisms that the brain needs to implement-including the fact that a certain level of abstraction, the fundamental combinatorial operation works on all categories alike (e.g., (Chomsky, 1995; Jackendoff, 2002). However, empirical investigations into 
natural language in real time suggest that combinatorial processing lead to multiple neural activation in different cortical regions, which cannot always be easily linked back to linguistic theory (Pylkkänen, 2019). This does not mean that certain phrasal constructions bypass syntactic information when not directly relevant - as already claimed in a previous work on adjectival modification and its relationship to the automaticity of syntax (Schell et al., 2017). Third, recent advancement in syntactic theory and preliminary behavioural studies, suggest that different phrases may have a much more complex internal structure than previously thought (Chesi and Canal, 2019; Zamparelli, 2014). Thus, future investigations should concentrate on systematic, within-category manipulations to explore phrasal and sentential combinations in more detail (Allen et al., 2012; Pylkkänen, 2020; Takashima et al., 2020).

\section{Conclusion}

In our study, we reported functional association between type of word combination and regional information in Broca's area. We believe that these findings pave the way for a finegrained characterisation of the linguistic features at work during combinatorial processes (Chesi and Canal, 2019), and they generally support spatiotemporal neural models showing that different neural populations might be recruited for different processing demands (Bernacchia et al., 2011).

\section{Acknowledgments}

The authors wish to thank Jöran Lipsien and Martin Hebart for their valuable insights on the data collection and the methodological analysis, and Joshua Grant for his comments on a previous version of the manuscript. The authors would also like to thank all participants who took part in the study. This work was founded by the Max Planck Society.

\section{Author contributions}

MS, ADF, and EZ conceptualized the study; MS collected the data; MS and EZ analyzed the data; MS, ADF and EZ wrote the manuscript; all authors discussed results, revised manuscript, and approved it for publication.

\section{Competing interests}

The authors declare that they have no conflict of interest.

\section{References}

Allen, K., Pereira, F., Botvinick, M., Goldberg, A.E., 2012. Distinguishing grammatical constructions with fMRI pattern analysis. Brain Lang. 123, 174-182. https://doi.org/10.1016/j.bandl.2012.08.005

Amici, S., Brambati, S.M., Wilkins, D.P., Ogar, J., Dronkers, N.L., Miller, B.L., Gorno-Tempini, M.L., 2007. Anatomical correlates of sentence comprehension and verbal working memory in neurodegenerative disease. J. Neurosci. 27, 6282-90. https://doi.org/10.1523/JNEUROSCI.1331-07.2007
Amunts, K., Schleicher, A., Burgel, U., Mohlberg, H., Uylings, H.B., Zilles, K., 1999. Broca's region revisited: cytoarchitecture and intersubject variability. J. Comp. Neurol. 412, 319-341.

Artoni, F., d'Orio, P., Catricalà, E., Conca, F., Bottoni, F., Pelliccia, V., Sartori, I., Russo, G. Lo, Cappa, S.F., Micera, S., Moro, A., 2020. High gamma response tracks different syntactic structures in homophonous phrases. Sci. Rep. 10, 7537. https://doi.org/10.1038/s41598-020-64375-9

Baker, M., 2003. Lexical categories - verbs, nouns and adjectives. Cambridge University Press, Cambridge.

Berkovitch, L., Dehaene, S., 2019. Subliminal syntactic priming. Cogn. Psychol. 109, 26-46. https://doi.org/10.1016/j.cogpsych.2018.12.001

Bernacchia, A., Seo, H., Lee, D., Wang, X.-J., 2011. A reservoir of time constants for memory traces in cortical neurons. Nat. Neurosci. 14, 366-372. https://doi.org/10.1038/nn.2752

Bernstein, J.B., 2008. Reformulating the Determiner Phrase Analysis. Lang. Linguist. Compass 2, 1246-1270. https://doi.org/10.1111/j.1749-818X.2008.00091.x

Bornkessel, I., Zysset, S., Friederici, A.D., von Cramon, D.Y., Schlesewsky, M., 2005. Who did what to whom? The neural basis of argument hierarchies during language comprehension. Neuroimage 26, 221-233. https://doi.org/10.1016/j.neuroimage.2005.01.032

Bradley, D.C., Garrett, M.F., 1983. Hemisphere differences in the recognition of closed and open class words. Neuropsychologia 21, 155-159. https://doi.org/10.1016/0028-3932(83)90082-9

Burroughs, A., Kazanina, N., Houghton, C., 2021. Grammatical category and the neural processing of phrases. Sci. Rep. 11. https://doi.org/10.1038/s41598-021-81901-5

Campbell, K.L., Tyler, L.K., 2018. Language-related domain-specific and domain-general systems in the human brain. Curr. Opin. Behav. Sci. 21, 132-137. https://doi.org/10.1016/J.COBEHA.2018.04.008

Chang, C.-C., Lin, C.-J., 2011. LIBSVM: A Library for Support Vector Machines. ACM Trans. Intell. Syst. Technol. 2, 1-27. https://doi.org/doi:10.1145/1961189.1961199

Chen, L., Goucha, T., Männel, C., Friederici, A.D., Zaccarella, E., 2021. Hierarchical syntactic processing is beyond mere associating: Functional magnetic resonance imaging evidence from a novel artificial grammar. Hum. Brain Mapp. 42, 3253-3268. https://doi.org/10.1002/hbm.25432

Chesi, C., Canal, P., 2019. Person Features and Lexical Restrictions in Italian Clefts. Front. Psychol. 10. https://doi.org/10.3389/fpsyg.2019.02105

Chiou, R., Humphreys, G.F., Jung, J.Y., Lambon Ralph, M.A., 2018. Controlled semantic cognition relies upon dynamic and flexible interactions between the executive 'semantic control' and hub-and-spoke 'semantic representation' systems. Cortex 103, 100-116. https://doi.org/10.1016/j.cortex.2018.02.018 
Chomsky, N., 1995. The Minimalist Program, Current Studies in Linguistics. MIT Press, Cambridge.

Del Prato, P., Pylkkanen, L., 2014. MEG evidence for conceptual combination but not numeral quantification in the left anterior temporal lobe during language production. Front Psychol 5, 524. https://doi.org/10.3389/fpsyg.2014.00524

Ding, N., Melloni, L., Zhang, H., Tian, X., Poeppel, D., 2016. Cortical tracking of hierarchical linguistic structures in connected speech. Nat. Neurosci. 19, 158-164. https://doi.org/10.1038/nn.4186

Eickhoff, S.B., Stephan, K.E., Mohlberg, H., Grefkes, C., Fink, G.R., Amunts, K., Zilles, K., 2005. A new SPM toolbox for combining probabilistic cytoarchitectonic maps and functional imaging data. Neuroimage 25, 1325-1335. https://doi.org/10.1016/j.neuroimage.2004.12.034

Etzel, J.A., Gazzola, V., Keysers, C., 2009. An introduction to anatomical ROI-based fMRI classification analysis. Brain Res. 1282, 114-125. https://doi.org/10.1016/J.BRAINRES.2009.05.090

Friederici, A.D., 2011. The brain basis of language processing: from structure to function. Physiol. Rev. 91, 1357-1392. https://doi.org/10.1152/physrev.00006.2011

Friederici, A.D., 1985. Levels of processing and vocabulary types: evidence from on-line comprehension in normals and agrammatics. Cognition 19, 133-166. https://doi.org/10.1016/0010-0277(85)90016-2

Friederici, A.D., 1983. Children's sensitivity to function words during sentence comprehension 21, 717-740. https://doi.org/10.1515/LING.1983.21.5.717

Friederici, A.D., Kotz, S.A., 2003. The brain basis of syntactic processes: functional imaging and lesion studies. Neuroimage 20, S8-17.

Friederici, A.D., Meyer, M., von Cramon, D.Y., 2000a. Auditory language comprehension: an event-related fMRI study on the processing of syntactic and lexical information. Brain Lang. 74, 289-300. https://doi.org/10.1006/brln.2000.2313

Friederici, A.D., Opitz, B., von Cramon, D.Y., 2000b. Segregating semantic and syntactic aspects of processing in the human brain: an fMRI investigation of different word types. Cereb. Cortex 10, 698-705.

Georgopoulos, A., Schwartz, A., Kettner, R., 1986. Neuronal population coding of movement direction. Science (80-. ). 233, 1416-1419. https://doi.org/10.1126/science.3749885

Goucha, T., Friederici, A.D., 2015. The language skeleton after dissecting meaning: A functional segregation within Broca's Area. Neuroimage 114, 294-302. https://doi.org/10.1016/j.neuroimage.2015.04.011

Goucha, T., Zaccarella, E., Friederici, A.D., 2017. A revival of Homo loquens as a builder of labeled structures: Neurocognitive considerations. Neurosci. Biobehav. Rev. 81, 213-224. https://doi.org/10.1016/j.neubiorev.2017.01.036
Graessner, A., Zaccarella, E., Hartwigsen, G., 2021. Differential contributions of left-hemispheric language regions to basic semantic composition. Brain Struct. Funct. https://doi.org/10.1007/s00429-020-02196-2

Hagoort, P., 2005. On Broca, brain, and binding: a new framework. Trends Cogn. Sci. 9, 416-423. https://doi.org/10.1016/j.tics.2005.07.004

Hamilton, A.C., Martin, R.C., Burton, P.C., 2009. Converging functional magnetic resonance imaging evidence for a role of the left inferior frontal lobe in semantic retention during language comprehension. Cogn. Neuropsychol. 26, 685-704. https://doi.org/10.1080/02643291003665688

Hartwigsen, G., Weigel, A., Schuschan, P., Siebner, H.R., Weise, D., Classen, J., Saur, D., 2016. Dissociating Parieto-Frontal Networks for Phonological and Semantic Word Decisions: A Condition-andPerturb TMS Study. Cereb. Cortex 26, 2590-2601. https://doi.org/10.1093/cercor/bhv092

Haufe, S., Meinecke, F., Görgen, K., Dähne, S., Haynes, J.-D., Blankertz, B., Bießmann, F., 2014. On the interpretation of weight vectors of linear models in multivariate neuroimaging. Neuroimage 87, 96-110. https://doi.org/10.1016/j.neuroimage.2013.10.067

Haynes, J.D., Rees, G., 2006. Decoding mental states from brain activity in humans. Nat. Rev. Neurosci. https://doi.org/10.1038/nrn1931

Hebart, M.N., Görgen, K., Haynes, J.-D., 2014. The Decoding Toolbox (TDT): a versatile software package for multivariate analyses of functional imaging data. Front. Neuroinform. 8, 88. https://doi.org/10.3389/fninf.2014.00088

Heim, I., 1982. The semantics of definite and indefinite NPs. University of Massachusetts at Amherst.

Heim, I., Kratzer, K., 1998. Semantics in Generative Grammar. Blackwell Publishers Ltd.

Herrmann, B., Obleser, J., Kalberlah, C., Haynes, J.D., Friederici, A.D., 2012. Dissociable neural imprints of perception and grammar in auditory functional imaging. Hum. Brain Mapp. 33, 584-595. https://doi.org/10.1002/hbm.21235

Hewitt, J., Liang, P., 2019. Designing and Interpreting Probes with Control Tasks, in: Proceedings of the 2019 Conference on Empirical Methods in Natural Language Processing and the 9th International Joint Conference on Natural Language Processing (EMNLP-IJCNLP). Association for Computational Linguistics, Stroudsburg, PA, USA. https://doi.org/10.18653/v1/D19-1275

Humphries, C., Binder, J.R., Medler, D.A., Liebenthal, E., 2006. Syntactic and semantic modulation of neural activity during auditory sentence comprehension. J. Cogn. Neurosci. 18, 665-679. https://doi.org/10.1162/jocn.2006.18.4.665

Humphries, C., Love, T., Swinney, D., Hickok, G., 2005. Response of anterior temporal cortex to syntactic and prosodic manipulations during sentence processing. Hum. Brain Mapp. 26, 128-138. 
https://doi.org/10.1002/hbm.20148

Jackendoff, R., 2002. Foundations of language: brain, meaning, grammar, evolution. Oxford University Press, Oxford.

Jefferies, E., Patterson, K., Jones, R.W., Lambon Ralph, M.A., 2009. Comprehension of concrete and abstract words in semantic dementia. Neuropsychology 23. https://doi.org/10.1037/a0015452

Jobard, G., Vigneau, M., Mazoyer, B., TzourioMazoyer, N., 2007. Impact of modality and linguistic complexity during reading and listening tasks. Neuroimage 34, 784-800. https://doi.org/10.1016/j.neuroimage.2006.06.067

Kamitani, Y., Sawahata, Y., 2010. Spatial smoothing hurts localization but not information: Pitfalls for brain mappers. Neuroimage 49, 1949-1952. https://doi.org/10.1016/j.neuroimage.2009.06.040

Magrassi, L., Aromataris, G., Cabrini, A., AnnovazziLodi, V., Moro, A., 2015. Sound representation in higher language areas during language generation. Proc. Natl. Acad. Sci. U. S. A. 112, 1868-73. https://doi.org/10.1073/pnas.1418162112

Makuuchi, M., Bahlmann, J., Anwander, A., Friederici, A.D., 2009. Segregating the core computational faculty of human language from working memory. Proc. Natl. Acad. Sci. U. S. A. 106, 8362-8367. https://doi.org/10.1073/pnas.0810928106

Matchin, W., Hammerly, C., Lau, E., 2017. The role of the IFG and pSTS in syntactic prediction: Evidence from a parametric study of hierarchical structure in fMRI. Cortex 88, 106-123. https://doi.org/10.1016/j.cortex.2016.12.010

Matchin, W., Hickok, G., $2019 . \quad$ The Cortical Organization of Syntax. Cereb. Cortex. https://doi.org/10.1093/cercor/bhz180

Mazoyer, B., Tzourio, N., Frak, V., Syrota, A., Murayama, N., O, L., Salamon, G., Dehaene, S., Cohen, L., Mehler, J., 1993. The cortical representation of speech. J. Cogn. Neurosci. 5, 467-479. https://doi.org/10.1162/jocn.1993.5.4.467

Mollo, G., Karapanagiotidis, T., Bernhardt, B.C., Murphy, C.E., Smallwood, J., Jefferies, E., 2016. An individual differences analysis of the neurocognitive architecture of the semantic system at rest. Brain Cogn. 109. https://doi.org/10.1016/j.bandc.2016.07.003

Musso, M., Moro, A., Glauche, V., Rijntjes, M., Reichenbach, J., Buchel, C., Weiller, C., 2003. Broca's area and the language instinct. Nat. Neurosci. 6, 774-781. https://doi.org/10.1038/nn1077

Nelson, M.J., El Karoui, I., Giber, K., Yang, X., Cohen, L., Koopman, H., Cash, S.S., Naccache, L., Hale, J.T., Pallier, C., Dehaene, S., 2017. Neurophysiological dynamics of phrase-structure building during sentence processing. Proc. Natl. Acad. Sci. U. S. A. 114, E3669-E3678. https://doi.org/10.1073/pnas.1701590114

Oldfield, R.C., 1971. The assessment and analysis of handedness: the Edinburgh inventory. Neuropsychologia 9,
97-113. https://doi.org/10.1016/0028-3932(71)900674

Pallier, C., Devauchelle, A.D., Dehaene, S., 2011. Cortical representation of the constituent structure of sentences. Proc. Natl. Acad. Sci. U. S. A. 108, 2522-2527. https://doi.org/10.1073/pnas.1018711108

Partee, B.H., 2010. Formal Semantics: Origins, Issues, Early Impact. Balt. Int. Yearb. Cogn. Log. Commun. 6. https://doi.org/10.4148/biyclc.v6i0.1580

Pouget, A., Dayan, P., Zemel, R., 2000. Information processing with population codes. Nat. Rev. Neurosci. 1, 125-132. https://doi.org/10.1038/35039062

Pulvermüller, F., Lutzenberger, W., Birbaumer, N., 1995. Electrocortical distinction of vocabulary types. Electroencephalogr. Clin. Neurophysiol. 94, 357-370.

Pylkkänen, L., 2020. Neural basis of basic composition: what we have learned from the red-boat studies and their extensions. Philos. Trans. R. Soc. B Biol. Sci. 375. https://doi.org/10.1098/rstb.2019.0299

Pylkkänen, L., 2019. The neural basis of combinatory syntax and semantics. Science (80-. ). https://doi.org/10.1126/science.aax0050

Rea, L.M., Parker, R.A., 1992. Designing and conducting survey research. Jossey-Bass, San Francisco, CA.

Rizzi, L., 2012. Core linguistic computations: How are they expressed in the mind/brain? J. Neurolinguistics 25, 489-499. https://doi.org/10.1016/j.jneuroling.2009.12.005

Rodd, J.M., Davis, M.H., Johnsrude, I.S., 2005. The neural mechanisms of speech comprehension: fMRI studies of semantic ambiguity. Cereb. Cortex 15, 1261-1269. https://doi.org/10.1093/cercor/bhi009

Rogalsky, C., Hickok, G., 2009. Selective attention to semantic and syntactic features modulates sentence processing networks in anterior temporal cortex. Cereb. Cortex 19, 786-796. https://doi.org/10.1093/cercor/bhn126

Sassenhagen, J., Alday, P.M., 2016. A common misapplication of statistical inference: Nuisance control with nullhypothesis significance tests. Brain Lang. 162, 42-45. https://doi.org/10.1016/j.bandl.2016.08.001

Schell, M., Zaccarella, E., Friederici, A.D., 2017. Differential cortical contribution of syntax and semantics: An fMRI study on two-word phrasal processing. Cortex 96, 105-120. https://doi.org/10.1016/j.cortex.2017.09.002

Shapiro, L.P., Jensen, L.R., 1986. Processing open and closed class-headed nonwords: left hemisphere support for separate vocabularies. Brain Lang. 28, 318-327. https://doi.org/10.1016/0093-934X(86)90108-2

Sheng, J., Zheng, L., Lyu, B., Cen, Z., Qin, L., Tan, L.H., Huang, M.-X., Ding, N., Gao, J.-H., 2019. The Cortical Maps of Hierarchical Linguistic Structures during Speech Perception. Cereb. Cortex 29, 3232-3240. https://doi.org/10.1093/cercor/bhy191

Stark, C.E.L., Squire, L.R., 2001. When zero is not zero: The problem of ambiguous baseline conditions in fMRI. Proc. Natl. Acad. Sci. 98. https://doi.org/10.1073/pnas.221462998 
Stowe, L.A., Paans, A.M., Wijers, A.A., Zwarts, F., Mulder, G., Vaalburg, W., 1999. Sentence comprehension and word repetition: a positron emission tomography investigation. Psychophysiology 36, 786-801. https://doi.org/10.1111/1469-8986.3660786

Takashima, A., Konopka, A., Meyer, A., Hagoort, P., Weber, K., 2020. Speaking in the brain: The interaction between words and syntax in sentence production. J. Cogn. Neurosci. 32, 1466-1483. https://doi.org/10.1162/jocn_a_01563

Tyler, L.K., Marslen-Wilson, W., 2008. Fronto-temporal brain systems supporting spoken language comprehension. Philos. Trans. R. Soc. B Biol. Sci. https://doi.org/10.1098/rstb.2007.2158

Vitello, S., Warren, J.E., Devlin, J.T., Rodd, J.M., 2014. Roles of frontal and temporal regions in reinterpreting semantically ambiguous sentences. Front. Hum. Neurosci. 8, 530. https://doi.org/10.3389/FNHUM.2014.00530

Wu, C.Y., Zaccarella, E., Friederici, A.D., 2019. Universal neural basis of structure building evidenced by network modulations emerging from Broca's area: The case of Chinese. Hum. Brain Mapp. 40, 1705-1717. https://doi.org/10.1002/hbm.24482

Wurm, M.F., Lingnau, A., 2015. Decoding actions at different levels of abstraction. J. Neurosci. 35, 7727-7735. https://doi.org/10.1523/JNEUROSCI.0188-15.2015

Yang, C., 2013. Ontogeny and phylogeny of language. Proc. Natl. Acad. Sci. U. S. A. 110, 6324-6327. https://doi.org/10.1073/pnas.1216803110

Zaccarella, E., Friederici, A.D., 2015. Merge in the human brain: A sub-region based functional investigation in the left pars opercularis. Front. Psychol. 6, 1818. https://doi.org/10.3389/fpsyg.2015.01818

Zaccarella, E., Meyer, L., Makuuchi, M., Friederici, A.D., 2017a. Building by Syntax: The Neural Basis of Minimal Linguistic Structures. Cereb. Cortex 27, 411-421. https://doi.org/10.1093/cercor/bhv234

Zaccarella, E., Schell, M., Friederici, A.D., 2017b. Reviewing the functional basis of the syntactic Merge mechanism for language: A coordinatebased activation likelihood estimation meta-analysis. Neurosci. Biobehav. Rev. 80, 646-656. https://doi.org/10.1016/j.neubiorev.2017.06.011

Zamparelli, R., 2014. Layers in the Determiner Phrase. Routledge. https://doi.org/10.4324/9781315054360

created with overleaf.com 09.02.2022 